\title{
Abnormality Detection in Ovarian Ultrasound Images using Active Contours
}

\author{
Usha B $S^{1}$, Sandya $S^{2}$ \\ ${ }^{1}$ Research Scholar (ECE, VTU), RNS Institute of Technology, Bengaluru, India \\ 2Nitte Meenakshi Institute of Technology, Bengaluru, India \\ bsusha@gmail.com, sandya9prasad@gmail.com
}

\begin{abstract}
Analyzing ovarian size parameters and its shape is the first hand information required in Ovary diagnosis. Automated segmentation of Ovary and measuring its size and analyzing its shape can help doctors in mass screening programme in faster diagnosis. In this paper, we propose a new improved algorithm (anisotropic diffusion filter, CLAHE enhancement, and active contour segmentation) to extract the Ovary from its homogeneous background followed by geometrical and shape feature extraction of the Ovary for automated computer-assisted analysis. The proposed algorithm is tested on 75 Transvaginal ultrasound images of ovaries. The experimental results are validated against the manual measurements done by the expert and the results obtained by our algorithm are in good agreement with experts inputs. The proposed algorithm could achieve an average Error Percentage $E_{1}$ of $4 \%$ for Major-Axis length and average Error Percentage $\mathrm{EM}_{2}$ of $5.43 \%$ for Minor-Axis length and classification rate of $91.66 \%$.
\end{abstract}

Keywords: Image segmentation, Ovary, anisotropic diffusion filter, active contour, Geometric features, Ovary classification

\section{INTRODUCTION}

Ultrasound imaging has become an integral diagnostic tool to evaluate the gynecological health of a woman [1]. In this paper, we propose a new modified algorithm for computerassisted analysis of the ultrasound images of women's ovaries. Ovary is scanned to diagnose abnormalities like infertility, irregular menstrual cycles, PCOS, cysts, tumors etc. Analyzing the ovarian size parameters and its shape are one of the first steps in diagnosis of the Ovary [13].The normal range of Ovary size parameters is listed in Table I which was obtained in literature and supported by our expert Dr. Sriprada Vinekar, Gynecologist and Sonographist. Lists the parameters considered to evaluate the shape of the Ovary. Values listed in Table 1 are used to evaluate whether Ovary is normal or abnormal. 
Table 1: Ovary Condition

\begin{tabular}{|l|l|l|}
\hline Normal Ovary & Min & Max \\
\hline Major-axis length M1 & $3 \mathrm{~cm}$ & $5 \mathrm{~cm}$ \\
\hline Minor-axis length M2 & $1.5 \mathrm{~cm}$ & $3 \mathrm{~cm}$ \\
\hline Thickness & $0.5 \mathrm{~cm}$ & $1.5 \mathrm{~cm}$ \\
\hline Area & $3.54 \mathrm{~cm}^{2}$ & $11.8 \mathrm{~cm}^{2}$ \\
\hline Perimeter & $7.45 \mathrm{~cm}$ & $12.95 \mathrm{~cm}$ \\
\hline Form Factor F & 0.8 & 0.88 \\
\hline Compactness Q & 14.2 & 15.6 \\
\hline Eccentricity E & $>0.5$ & \\
\hline Shape & Oval (Almond) \\
\hline
\end{tabular}

\section{BACKGROUND WORK}

Automated measurement of ovarian parameters requires segmentation of the Ovary from the ovarian ultrasound images. The ultrasound images are of low contrast and poor quality due to the presence of speckle noise. Moreover the Region of Interest (Ovary) is highly homogeneous with its background. Hence, edge preserving speckle filters are required to improve the image quality and possibly the diagnostic potential of the image. In [1] Bilateral filter proposed by Tomasi C, Manduchi R[4] was used for speckle removal, Contrast Stretching and Power Law Transformation were used for enhancement, adaptive Thresholding and Global Thresholding for segmentation to extract Ovary. The average Error Percentage for Major-Axis length $\left(E_{M 1}\right)$ obtained was $11.9 \%$ and the average Error Percentage for Minor-Axis length ( $\left.E_{M 2}\right)$ obtained was $17.4 \%$. In [2] anisotropic diffusion filter proposed by Perona and Malik [5, 6] was used for speckle reduction, CLAHE [7] followed by adaptive binary thresholding to extract Ovary. The algorithm could achieve an average Error Percentage of 5.27\% for Major-Axis length $\left(E_{M 1}\right)$ and average Error Percentage of $6.1 \%$ for Minor-Axis length $\left(E_{M 2}\right)$. In [8, 9] active contours was used for detection of follicles. The average detection rate was $96.66 \%$, false rejection rate (FRR) $3.33 \%$, and false acceptance rate (FAR) $15.04 \%$. Active contours detect edges even in high noisy region and hence in this paper we propose to use active contours for segmentation.

The paper is organized as follows: Section III provides the description of the algorithm where speckle reduction filter, contrast enhancement methods, segmentation techniques and feature extraction are explained in detail. Section IV, the experimental results are discussed along with manual measurements done by the expert and section $\mathrm{V}$ contains conclusions and future scope of work. 


\section{Measurement Of Ovarian Features AND Classification}

The input is a Transvaginal ultrasound image of Ovary acquired from GE-LOGIQ Book XP ultrasound machine which is a high performance multipurpose color hand-carried imaging system. The images are obtained in JPEG-LS (24-bit, 532×434×3) format. The sample ultrasound image is shown in Figure. 1 consists of the Ovary region and Ovarian follicles inside the Ovary.

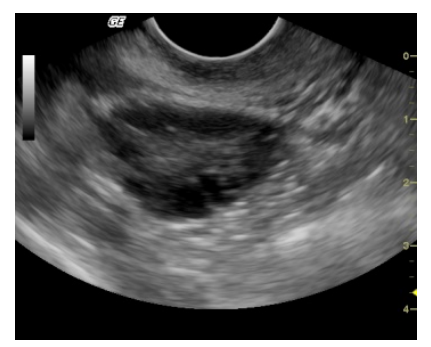

Figure. 1 Ultrasound Ovary image

The input image has identical RGB planes hence, only R-plane (8-bit, 532×434) is considered as the input image. The position of the Region of Interest, Ovary in all images is in the center of the fan area, so a sub-image $I_{\text {ROI }}$ containing only the Ovary (ROI) of size [310 420] is extracted.

\subsection{Preprocessing}

The proposed Ovary feature extraction system is shown in Figure.2. Measurement of the size and shape features of the Ovary requires segmentation of the Ovary from the ultrasound image. Since ultrasound images are invariably noisy due to speckle noise, we use speckle reducing anisotropic diffusion filter for denoising. The edge magnitude parameter $k$ was chosen as 0.9 and number of iterations fixed at 5 . The filtered output $\mathrm{I}_{\mathrm{f} 2}$ has very good mean preservation, variance reduction, and edge localization.

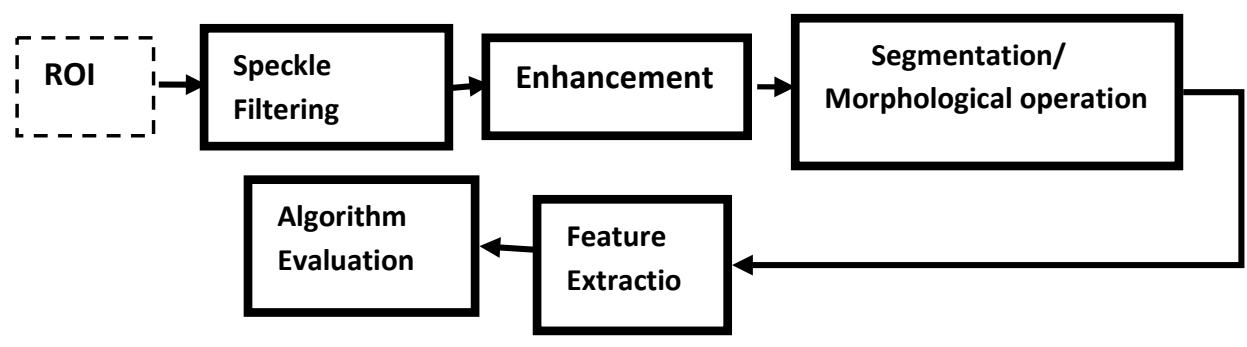

Figure.2 Ovary Feature Extraction System

To create a good contrast between the Ovary and the background which is essential for segmentation, Contrast Limited Adaptive Histogram Equalization (CLAHE) is applied. This algorithm operates on small data regions (tiles), instead of an entire image. Each tile's contrast is enhanced using Rayleigh's distribution. The neighboring tiles are combined using bilinear interpolation to eliminate artificially induced boundaries. In this algorithm we have chosen the tile value as [3 2], each tile's contrast is enhanced using Rayleigh's distribution. The clip-limit is 
chosen as 0.1 , range as original and alpha which is a distribution parameter is chosen as 6 . The output of the enhancement stage $\operatorname{ICL}(x, y)$ has a clear contrast between Ovary and background and is suitable for segmentation.

\subsection{Processing}

The processing stage has Segmentation, Morphological operation and feature extraction. The active contours without edges are used for segmentation. The resulting image after segmentation has many regions apart from Ovary. To begin with regions touching the image border are removed followed by region filling operation. We next perform morphological erosion ( 3 times with window size $3 \times 3$ ) followed by morphological dilation ( 3 times with window size $3 \times 3$ ). Many small phantom regions exist along with the Ovary region. A connected component labeling algorithm finds all connected components in the image and assigns a unique label to all points in the same component. Regions having smaller area than the threshold $\mathrm{T}$ are removed. This might result with more than one component. The Centroid $(\mathrm{C}=$ $\mathrm{Cx}, \mathrm{Cy}$ ) of the remaining components are found. In the current database, the Ovary is in the middle of the fan area and the mean centroid $(C=C x, C y)$ of Ovary is experimentally found. Rule1 \& Rule2 shown below are used to retain Ovary and eliminate the rest of the regions/components.

Rule1: $\mu C x-k \sigma C x \leq C x \leq \mu C x+k \sigma C x$

Rule2: $\mu \mathrm{Cy}-\mathrm{k} \sigma \mathrm{Cy} \leq \mathrm{Cy} \leq \mu \mathrm{Cy}+\mathrm{k} \sigma \mathrm{Cy}$

\subsection{Feature Extraction}

The outcome of the processing stage is a single component, Ovary. The sample input Ovary and the segmentation result is shown in Figure.3.
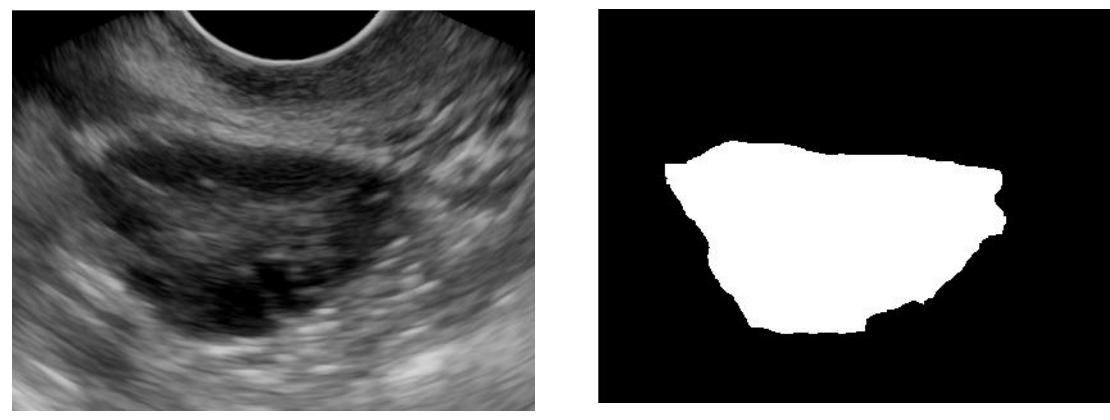

Figure.3 Segmentation result of Ovary

The geometrical and shape features of the segmented region are extracted which are grouped under F1 and F2 feature sets. The feature set F1: is the size based features and F2: is the shape based features [3].

F1: This feature set includes the Major-axis length M1, Minor-axis length M2, Area A and Perimeter $P$. 
F2: This feature set includes Form Factor F, Compactness Q, and Eccentricity E.

\subsection{Classification}

In the training phase, the F1 and F2 feature sets of the ovaries are extracted. The mean and standard deviation of each of this parameter is calculated. The $\mu$ and $\sigma$ of these parameters are listed in TABLE 2. Based on these values classification rules are formulated as shown in TABLE 3.

Table 2: mean and Standard deviations

\begin{tabular}{|c|c|}
\hline Parameter & Mean, sigma \\
\hline$\mu \mathrm{M}_{1}, \sigma \mathrm{M}_{1}$ & $3.459,0.757$ \\
\hline$\mu \mathrm{M}_{2}, \sigma \mathrm{M}_{2}$ & $1.987,0.565$ \\
\hline$\mu \mathrm{A}, \sigma \mathrm{A}$ & $5.494,2.5936$ \\
\hline$\mu \mathrm{P}, \sigma \mathrm{P}$ & $10.28,2.82$ \\
\hline$\mu \mathrm{F}, \sigma_{\mathrm{F}}$ & $0.8451,0.12$ \\
\hline$\mu \mathrm{Q}, \sigma_{\mathrm{Q}}$ & $15.15,2.97$ \\
\hline$\mu \mathrm{C}_{\mathrm{x}}, \sigma_{\mathrm{Cx}}$ & $191.32,37.65$ \\
\hline$\mu \mathrm{C}_{y}, \sigma_{\mathrm{Cy}}$ & $110.77,27.277$ \\
\hline
\end{tabular}

The classification rules of the $\mathrm{k} \sigma$-Classifier are formulated as in TABLE IV. A region is classified as a normal Ovary, if the following conditions Rulei, $i=1,2, \ldots . .9$ are satisfied. The value of constant $\mathrm{k}$ is $2, \alpha$ is 0.3 in the experiment. All the values are empirically determined.

Table 3: Rules

Rule1: $\mu C x-k \sigma C x \leq C x \leq \mu C x+k \sigma C x$

Rule2: $\mu \mathrm{Cy}-\mathrm{k} \sigma \mathrm{Cy} \leq \mathrm{Cy} \leq \mu \mathrm{Cy}+\mathrm{k \sigma Cy}$

Rule3: $\mu M_{1}-k / 3 \sigma M_{1}+\alpha \leq M_{1} \leq \mu M_{1}+k \sigma M_{1}+\alpha$

Rule4: $\mu \mathrm{M}_{2}-\mathrm{k} / 3 \sigma \mathrm{M}_{2}+\alpha_{\leq} \mathrm{M} 2 \leq \mu \mathrm{M}_{2}+k \sigma \mathrm{M}_{2}+\alpha$

Rule5: $\mu \mathrm{A}-\mathrm{k} / 2 \sigma \mathrm{A}+\alpha \leq \mathrm{A} \leq \mu \mathrm{A}+\mathrm{k} \sigma \mathrm{A}+2 \alpha$

Rule6: $\mu \mathrm{P}-\mathrm{k} / 2 \sigma \mathrm{P}+\alpha \leq \mathrm{P} \leq \mu \mathrm{P}+\mathrm{k} \sigma \mathrm{P}+\alpha$

Rule7: $\mu \mathrm{F}-\mathrm{k} \sigma_{\mathrm{F}} \leq \mathrm{F} \leq \mu \mathrm{F}+\mathrm{k} \sigma_{\mathrm{F}}$

Rule8: $\mu \mathrm{Q}-\mathrm{k} \sigma_{\mathrm{Q}} \leq \mathrm{Q} \leq \mu \mathrm{Q}+\mathrm{k} \sigma_{\mathrm{Q}}$

Rule9: $\mu \mathrm{E}-\mathrm{k} \sigma_{\mathrm{E}} \leq \mathrm{E} \leq \mu \mathrm{E}+\mathrm{k} \sigma_{\mathrm{E}}$ 
In the testing phase, Feature set F1 and F2 are computed for the segmented region of Ovary and the classification rules Rulei are applied to determine whether the Ovary is normal or not. Rule1 \& Rule2 1 are applied to retain Ovary and eliminate other regions when multiple regions of same size are the segmentation outcome. Rule3 to Rule6 classifies the Ovary based on size and are the key rules for classifying. R8 to R9 are based on shape and support the Rule3-6.

\subsection{Performance Measures}

The accuracy of Ovary segmentation is measured using two parameters- the error percentage $[1,2]$ for Major-axis length (EM1) and Minor-axis length (EM2) which is used to evaluate and validate the algorithm. We calculate the percentage error between the expert measured value of $\mathrm{M} 1$ and $\mathrm{M} 2$ and algorithm evaluated value of $\mathrm{M} 1$ and $\mathrm{M} 2$. This parameter qualifies the algorithm as to how best or how near the algorithm is to the expert measured values. It is required that ideally the error percentage is "ZERO" but a $\pm 12 \%$ deviation is acceptable as per the expert.

The error percentage [1, 2] for Major-axis length (EM1) and Minor-axis length (EM2) is calculated by using (1) and (2).

$$
\begin{aligned}
& \mathrm{E}_{\mathrm{M} 1}=\frac{\mid\left((\text { length })_{\text {obtained value }}-(\text { length })_{\text {Expert value }}\right) \mid}{(\text { length })_{\text {Expert value }}} \times 100 \\
& \mathrm{E}_{\mathrm{M} 2}=\frac{\mid\left((\text { width })_{\text {obtained value }}-(\text { width })_{\text {Expert value }) \mid} \mid\right.}{(\text { width })_{\text {Expert value }}} X 100
\end{aligned}
$$

Ovary segmentation process, might result in more than one regions. So in order to select the Ovary we use Rule $1 \& 2$. But if there are more than one regions with in the centroid with allowable variance, algorithm might select a wrong region as Ovary. This might lead to misclassification. An Ovary is classified either as normal or abnormal. Consequently, there are four events; two classifications and two misclassifications.

\subsubsection{Classification Rate}

The classification rate or classification accuracy is estimated by the ratio of the total number of correctly classified Ovary images (sum of true positives and true negatives) by the number of input Ovary images.

$$
\text { Classification Rate }=\frac{\# \text { of Correctly Classified Ovary Images }}{\# \text { of Input Ovary Images }}
$$




\subsubsection{False Acceptance Rate}

False acceptance, also called a type II error, is defined as the percentage of abnormal Ovary images classified as normal.

\subsubsection{False Rejection Rate}

False Rejection Ratio, also called a type I error, is defined as the percentage of normal Ovary images misclassified as abnormal.

\section{RESULTS AND DISCUSSIONS}

The database consists of 75 Transvaginal ultrasound images of ovaries. The size parameter $\mathrm{M} 1$ and $\mathrm{M} 2$ are provided by the expert for all the 75 images. The classification output is validated against the expert inputs. In our experiment, 40 images are used in training phase and 35 (20 normal, 15 abnormal) in testing phase. The sample outputs where the edge of the segmented Ovary is superimposed on the input image for subjective validation by the expert are shown in Fig 4. The first column of images shows the expert measured values of Major-axis length $\mathrm{M} 1$ and Minor axis length $\mathrm{M} 2$ and the second column shows the output images. The F1 features and F2 features and classification output of the sample images are shown in Table VI. The average Error Percentage obtained is 4\% for Major-Axis length (EM1) and 5.45\% for MinorAxis length (EM2) which is very much lesser than the BCAT method or ACAT method [3]. EM1 was $11.9 \%$ and $\mathrm{EM} 2$ was $17.4 \%$ using BCAT method whereas EM1 was $5.27 \%$ and $\mathrm{EM} 2$ was $6.1 \%$ using ACAT method. The average Error percentage using our new algorithm is very insignificant and proves to be better than ACAT and BCAT method.

The K- $\sigma$ classifier rules listed in TABLE IV is applied to the feature set F1, F2 to classify the Ovary as normal or abnormal. Out of 35 images used for testing, 20 images are normal and 15 images are abnormal. Classification results obtained by the algorithm is discussed below:

Among 20 normal Ovary images 18 are classified as normal and 2 are classified as abnormal. Among 15 abnormal images, 14 are classified as abnormal and 1 image is classified as normal. The classification rate is $91.66 \%$ and false acceptance rate (FAR) is $6.66 \%$ and false rejection rate (FRR) is $10 \%$ respectively.

The classification rate has significantly improved to $91.66 \%$ with our new algorithm compared to BCAT method in which classification rate was $76.67 \%$ and ACAT method in which classification rate was $85.8 \%$. The TABLE 4 shows the classification results. 
Table 4: Resuts

\begin{tabular}{|l||c|c|c|}
\hline Methodology & $\begin{array}{c}\text { Classification } \\
\text { Rate }\end{array}$ & $\begin{array}{c}\text { Type II } \\
\text { Error (FAR) }\end{array}$ & $\begin{array}{c}\text { Type I } \\
\text { Error( FRR) }\end{array}$ \\
\hline \hline New Algorithm & $91.66 \%$ & $6.66 \%$ & $10 \%$ \\
\hline BCAT & $76.67 \%$ & $26.66 \%$ & $20 \%$ \\
\hline ACAT & $85.8 \%$ & $13.33 \%$ & $15 \%$ \\
\hline
\end{tabular}

\section{CONCLUSIONS}

The classification rate depends on the choice of filter used, enhancement methods and segmentation method. The average Error Percentage is $4 \%$ for EM1 and $5.45 \%$ for EM2 which is significantly better than BCAT and ACAT method. From TABLE 5 it can be inferred that ACA has a good classification rate of $91.66 \%$, FAR of $6.66 \%$ and FRR of $10 \%$. The algorithm can be further improved using filters that can preserve edges better and segmentation methods that can extract the Ovary.

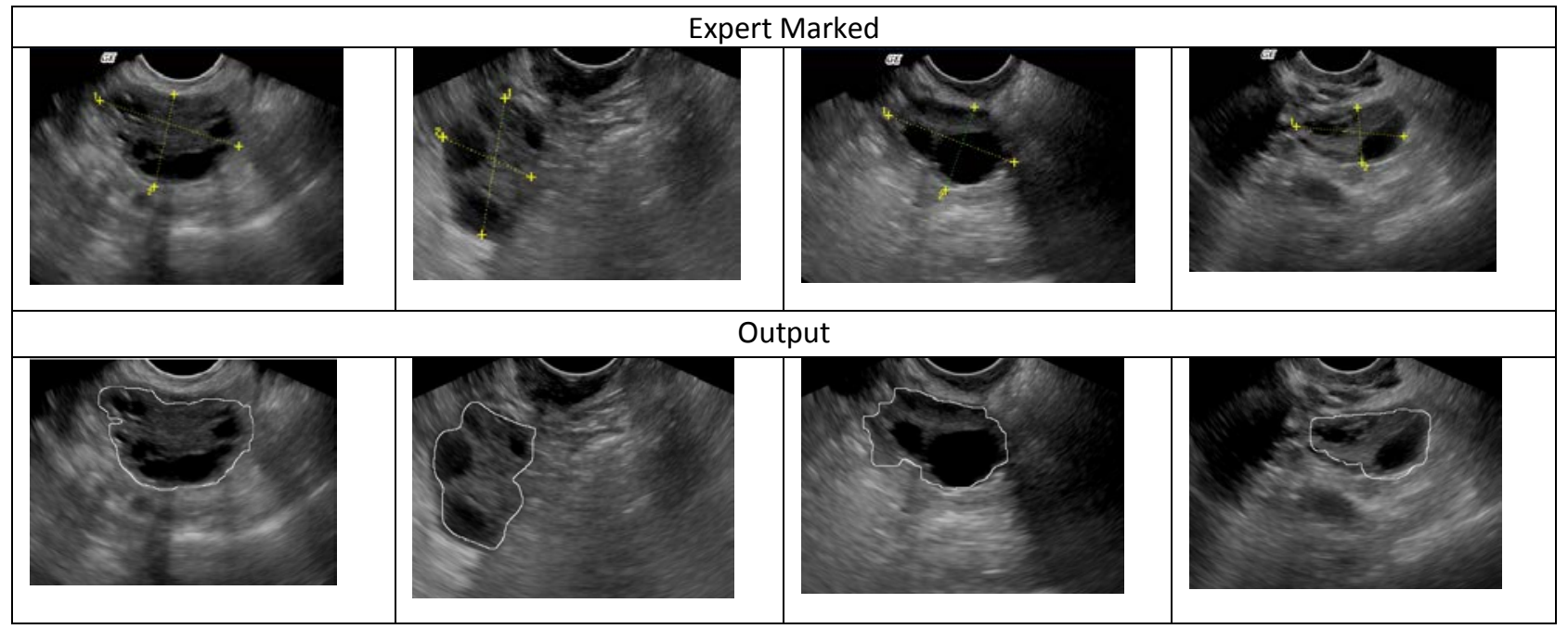

Figure 4. Segmentation results 
Table 5: Classification Rates

\begin{tabular}{|l|l|c|l|l|l|l|l|l|l|l|}
\hline$\#$ & $\mathrm{M}_{1}$ (Expert) & $\mathrm{M}_{2}$ (Expert) & $\mathrm{M}_{1}(\mathrm{Cm})$ & $\mathrm{M}_{2}(\mathrm{Cm})$ & $\mathrm{A}\left(\mathrm{Cm}^{2}\right)$ & $\mathrm{P}(\mathrm{Cm})$ & $\mathrm{F}$ & $\mathrm{Q}$ & $\mathrm{E}$ & Classification \\
\hline 1 & 2.84 & 1.41 & 2.838 & 1.443 & 3.14 & 6.75 & 0.86 & 14.51 & 0.86 & Normal \\
\hline 2 & 4.08 & 1.14 & 4.220 & 1.33 & 3.65 & 9.82 & 0.45 & 16.52 & 0.949 & Normal \\
\hline 3 & 3.04 & 1.7 & 3.073 & 1.749 & 4.19 & 7.83 & 0.85 & 14.6 & 0.8221 & Normal \\
\hline 4 & 3.04 & 1.7 & 2.936 & 1.645 & 3.77 & 7.45 & 0.85 & 14.72 & 0.8283 & Normal \\
\hline 5 & 2.81 & 2.1 & 2.771 & 2.030 & 4.41 & 7.62 & 0.95 & 13.16 & 0.6806 & Normal \\
\hline 6 & 4.14 & 1.17 & 4.202 & 1.134 & 3.72 & 9.66 & 0.5 & 25 & 0.9692 & Normal \\
\hline 7 & 4.1 & 3.32 & 4.0155 & 3.2479 & 10.17 & 11.45 & 0.97 & 12.89 & 0.7729 & Normal \\
\hline 8 & 2.9 & 1.82 & 2.8902 & 1.6936 & 3.83 & 7.43 & 0.87 & 14.41 & 0.8343 & Normal \\
\hline 9 & 2.38 & 1.9 & 2.4184 & 1.7017 & 3.21 & 6.55 & 0.94 & 13.36 & 0.7819 & Normal \\
\hline 1 & 6.24 & 4.27 & 6.2 & 3.97 & 18.4 & 20.06 & 0.57 & 21.83 & 0.7 & Abnormal \\
\hline 0 & & & 8 & & 3 & & & & 7 \\
\hline
\end{tabular}

\section{REFERENCES}

[1]. Usha B S, Sandya S, Shruthi G, "Size and Shape Based Ovarian Abnormality Detection of Ultrasound Images ",ICERECT-12, LNEE, Springer, 10.1007/978-81-322-1157-0_31

[2]. Usha B S, Sandya S, Measurement of Ovarian Size and Shape Parameters, IEEE Xplore, 10.1109/INDCON.2013.6726079

[3]. Usha B S, Sandya S, Ultrasound Ovary Image Classification Using Ko-Classifier, BME-2014, Ho Chi Minh City, Vietnam.

[4]. Carlo Tomasi and Roberto Manduchi, "Bilateral filtering for gray and color images," in Computer Vision, 1998. Sixth International Conference on . IEEE, 1998, pp. 839-846.

[5]. Pietro Perona and Jitendra Malik (July 1990). "Scale-space and edge detection using anisotropic diffusion". IEEE Transactions on Pattern Analysis and Machine Intelligence, 12 (7): 629-639. doi:10.1109/34.56205.

[6]. Y. Yu, S. T. Acton, and S. Member, "Speckle Reducing Anisotropic Diffusion," IEEE Transactions On Image Processing, vol. 11, no. 11, pp. 1260-1270, 2002, 88DOI 10.1109/TIP.2002.804276

[7]. Zuiderveld, Karel. "Contrast Limited Adaptive Histograph Equalization." Graphic Gems IV. San Diego: Academic Press Professional, 1994. 474-485.

[8]. P.S.Hiremath and Jyothi R. Tegnoor, Follicle Detection in Ultrasound Images of Ovaries using Scanline Thresholding Method 
Usha B.S. and Sandya S.; Abnormality Detection in Ovarian Ultrasound Images using Active Contours, Journal of Biomedical Engineering and Medical Imaging, Volume 1, Issue 4, Aug (2014), PP 14-23

[9]. P.S.Hiremath and Jyothi R. Tegnoor, "Recognition of Follicles in Ultrasound Images of Ovaries using Geometric Features" IEEE Transactions On Medical Imaging Vol. 17, No. 6 978-1-4244-4764. 